\title{
Professor Fritz Hubertus Kemper verstorben
}

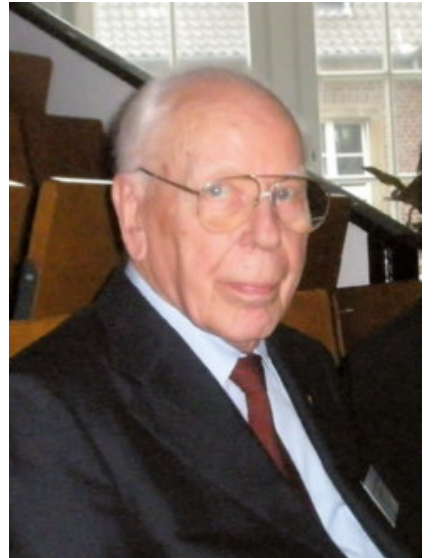

Die Gesellschaft für Phytotherapie trauert um Professor em. Dr. med. Dres. h.c. Fritz Hubertus Kemper, der am 28. März 2017 nach langer Krankheit in seinem 91. Lebensjahr verstorben ist.

Fritz Hubertus Kemper wurde am 9. Februar 1927 in Köln geboren. Nach dem Abitur 1944 studierte er in Köln und Bonn Medizin und promovierte 1951 an der Medizinischen Fakultät der Universität zu Köln. Danach begann er seine internistische Weiterbildung, zunächst in Lüdenscheid, ab 1952 in Frankfurt/Main. In Frankfurt verfasste er wissenschaftliche Arbeiten auf dem Gebiet der Kreislauf- und Hochdruckforschung und der Endokrinologie.

Nach Erlangung des Facharztes für Innere Medizin im Jahr 1956 ging er zu Prof. Dr. med. Dr. phil. Arnold Loeser an das Pharmakologische Institut der Westfälischen Wilhelms Universität Münster/ Westfalen. Dort arbeitete er zu pharmakologischen Wirkungen von Arzneipflanzen und deren Inhaltsstoffen. Er habilitierte sich 1958 mit der Schrift: „Experimentelle Grundlagen für eine therapeutische Anwendung von Lithospermum officinale zur Blockierung von Hormonen des Hypophysenvorderlappens" und erhielt die Venia legendi für das Gebiet Pharmakologie und Toxikologie. Im gleichen Jahr erhielt er auch die Anerkennung als Facharzt für Pharmakologie und Toxikologie sowie für Klinische Pharmakologie. Während dieser Zeit erlangte er grundlegende Erkenntnisse über die Wirkung von Arzneipflanzen, die entscheidend für seine weitere berufliche Zielsetzung wurden. Zugleich befasste er sich mit toxikologischen Fragestellungen. So publizierte er 1962 einen Beitrag zum schädigenden Einfluss von Thalidomid (Contergan) auf die endokrine Entwicklung von Hähnchen. 1964 wurde er außerplanmäßiger Professor, ab 1965 Wissenschaftlicher Rat und Professor an der Universität Münster. 1969 wurde er auf den dortigen Lehrstuhl für Pharmakologie und Toxikologie berufen und übernahm 1970 die Leitung des Instituts. Danach begann er zusammen mit seiner Mitarbeiterin Hilke Winterhoff maßgebliche Untersuchungen zur endokrinologischen Wirkung von Arzneipflanzen. Fritz Kemper war Dekan der Medizinischen Fakultät der Universität Münster von 1970 und 1971 und von 1984 und 1985. Danach war er hauptamtlich Geschäftsführender Ärztlicher Direktor der Medizinischen Einrichtungen in Münster bis zu seinem Ruhestand im Jahr 1993.

Die Geschichte der Umweltprobenbank für Human-Organproben an der Medizinischen Fakultät Münster begann 1974, als das Bundesinnenministerium bei Fritz Kemper nach Informationen zur Schwermetallbelastung der Deutschen fragte. Da hierzu keine statistischen Daten existierten, initialisierte er ein Pilotprojekt. Dieses wuchs mit der Zeit zu ei- ner festen Bundeseinrichtung heran, die heutzutage als wichtiges wissenschaftliches Instrument und als Grundlage für umweltpolitische Entscheidungen weltweit anerkannt ist. Er leitete die Umweltprobenbank bis 2006.

Seine große berufspolitische Begabung zeigte sich schon früh. Bereits seit 1968 war er Dauervertreter der Medizinischen Fakultät der Universität Münster beim Medizinischen Fakultätentag, ab 1973 war er Mitglied des Präsidiums und von 1985 bis 1997 dessen Präsident. Dieses Amt übte er in vollem Vertrauen der Medizinischen Fakultäten mit jeweils einstimmiger Wiederwahl in den Jahren 1988, 1991 und 1994 über vier Wahlperioden bis 1997 mit hohem Engagement und großem persönlichen Einsatz aus. Dank seiner fundierten Kenntnisse, seiner Erfahrungen als Hochschullehrer und seiner Durchsetzungskraft gelang es ihm, auch widerstrebende Interessen zu überbrücken und sachbezogene Entscheidungen herbeizuführen. Ein großer persönlicher Erfolg war die rasche $\mathrm{Zu}$ sammenführung der medizinischen Ausbildung in West- und Ostdeutschland im Jahr 1990.

Ein besonderes Anliegen war ihm die Weiterentwicklung der Approbationsordnung für Ärzte. Er setzte sich mit Nachdruck dafür ein, patientennahe Problemkreise aus Klinik und Praxis in die medizinische Hochschulausbildung zu integrieren und eine Begegnung der praktizierenden Ärzteschaft mit Vertretern und Institutionen der Hochschulmedizin auf der Basis gegenseitigen Verständnisses und Vertrauens zu ermöglichen. So war es u.a. seiner Initiative zu verdanken, dass der Querschnittsbereich 12 (Rehabilitation, Physikalische Medizin, Naturheilverfahren) in die Approba- 
tionsordnung ab 2002 aufgenommen wurde. Dadurch konnten Medizinstudenten im Rahmen dieser Pflichtveranstaltung u.a. auch in wissenschaftlicher Phytotherapie ausgebildet werden. Da er bereits ab dem Jahr 1996 über viele Jahre zusammen mit Prof. Malte Bühring, Berlin, die wissenschaftliche Loseblattsammlung „Naturheilverfahren und Unkonventionelle Medizinische Richtungen“ herausgab mit dem Ziel eines wissenschaftlichen Umgangs mit diesen Therapieverfahren, konnten die Unterrichtsveranstaltungen des QB12, insbesondere die Naturheilverfahren, von dem inzwischen umfangreichen Material sehr profitieren. Bis Anfang des neuen Jahrtausends war es nämlich kaum möglich, wissenschaftliche Arbeiten mit einem naturheilkundlichen bzw. komplementären Thema in internationalen Zeitschriften zu publizieren.

In Expertenkommissionen des Instituts für Medizinische und Pharmazeutische Prüfungsfragen (IMPP), Mainz, hat er sich für die Weiterentwicklung der „Akademischen Prüfungen“ eingesetzt. Seine erfolgreiche Arbeit als Vorsitzender der Kontroll-Kommission des IMPP verdient eine besondere Würdigung.

Von 1989 bis 2005 war er Mitglied in der Kammerversammlung der Ärztekammer Westfalen-Lippe. Er arbeitete u. a. im Ausschuss Hochschule / Ärztliche Ausbildung und im Ausschuss Umweltmedizin. In der Akademie für ärztliche Fortbildung der Ärztekammer und der Kassenärztlichen Vereinigung des Bezirks Westfalen-Lippe war er maßgeblich an der Gestaltung der Kurse „Umweltmedizin“ beteiligt und von 2000 bis 2007 Mitglied des Sektionsvorstands Hygiene und Umweltmedizin. Noch nach seinem Ruhestand erwarb er 1996 die Zusatzqualifikation „Umweltmedizin“. Er arbeitete zudem in den Ausschüssen „Ausbildung zum Arzt/Hochschule“ und in der „Ständigen Konferenz Krankenhaus“ der Bundesärztekammer mit.

Fritz Hubertus Kemper war für mehr als 25 Jahren Mitglied mehrerer Ständiger Kommissionen des Bundesgesundheitsamtes Berlin und dessen Nachfolgeorganisationen, wie des Bundesinstitutes für gesundheitlichen Verbraucher- schutz und des Bundesinstitutes für Arzneimittel und Medizinprodukte. Er war Gründungsmitglied in der Kosmetik-Kommission und über zehn Jahre Vorsitzender des Wissenschaftlichen Beirates des Bundesinstituts für gesundheitlichen Verbraucherschutz und Veterinärmedizin (BgVV). Als Sachverständiger auf dem Gebiet eines wissenschaftlich begründeten Verbraucherschutzes war er über Jahrzehnte Mitglied der Senatskommissionen der Deutschen Forschungsgemeinschaft.

Mehr als 20 Jahre vertrat er die Bundesrepublik Deutschland bei der Europäischen Union (EU) in einer Reihe von Gremien. So war er über zehn Jahre Vorsitzender des Wissenschaftlichen Ausschusses der EU zur Prüfung der Toxizität und Ökotoxizität chemischer Verbindungen (DG V). Seit 1990 war er Mitglied des Scientific Committee on Cosmetic Products and Non-Food Products (SCCNFP). Im Jahr 1996 übernahm er den Gründungsvorsitz des höchsten wissenschaftlichen Leitgremiums der EU, des Multidisciplinary Scientific Committee (MDSC).

Seine Leistungen für die Phytotherapie sollen nun noch genauer dargestellt werden. Mit seinen ab 1970 durchgeführten Untersuchungen untermauerte er die inzwischen allgemein akzeptierte Erkenntnis, dass Phytopharmaka als Vielstoffgemische mit teilweise pharmakologisch aktiven Inhaltsstoffen sich zwar in ihrer Zusammensetzung von synthetischen Arzneimitteln unterscheiden, dass aber ihre Wirkung wissenschaftlich zu begründen ist und zwar im Sinne einer - wie wir heute sagen - „multi-targetTherapie“. Dem seit Ende der 1960er Jahre dominierenden Dogma, dass es ein „single bullet“ für die Therapie einer jeden Erkrankung geben könne, ist er nicht gefolgt. Er vertrat er auch mit als erster die Ansicht, dass die Wirkung, die Wirksamkeit und die Unbedenklichkeit von Phytopharmaka mit vergleichbaren oder identischen Methoden wie für chemischsynthetische Arzneimittel belegt werden kann und muss.

Er hat viele Jahre der Kommission E des Bundesgesundheitsministeriums angehört, hat sich aber auch sehr frühzeitig für die Akzeptanz der Phytopharmaka in Europa eingesetzt. Die Gründung der "European Scientific Cooperative on Phytotherapy“ (ESCOP) im Jahr 1989 als europäischer Dachverband nationaler Gesellschaften für Phytotherapie ist vor allem auf seine Initiative zurückzuführen. Als Chairman des Board of Directors von 1992 bis 2010 hat er die ESCOP maßgeblich geprägt und zu ihrer internationalen Akzeptanz beigetragen. Er hat dafür gesorgt, dass die Vertrauensmängel in die wissenschaftliche Phytotherapie auf der europäischen Ebene abgebaut wurden. Dies hat in der Folge zur Einrichtung des permanenten Ausschusses für pflanzliche Arzneimittel (HMPC) bei der Europäischen Arzneimittelagentur (EMA) geführt. Auch für die World Health Organisation (WHO) war er lange ständiger Berater auf dem Gebiet der Phytotherapie.

Fritz Hubertus Kemper war von 19922008 Präsident der Gesellschaft für Phytotherapie (GPT) und langjähriger Herausgeber der Zeitschrift für Phytotherapie. Er hat sich stets für eine wissenschaftlich begründete Therapie mit Phytopharmaka eingesetzt. Sein Name ist deshalb untrennbar mit dem Begriff „wissenschaftliche Phytotherapie“ verbunden.

Einer seiner letzten großen Erfolge gelang ihm im November 2013. Schon stark von seiner langjährigen Krankheit gezeichnet, erreichte er mit mir gemeinsam, dass die Gesellschaft für Phytotherapie in die Arbeitsgemeinschaft der wissenschaftlichen medizinischen Fachgesellschaften (AWMF) aufgenommen wurde. Diese Aufnahme war alles andere als selbstverständlich, da sie von den Mitgliedern der AWMF allgemein als komplementärmedizinische Fachgesellschaft betrachtet wurde. Seither finden Phytopharmaka mit belegter Wirksamkeit in den interdisziplinären medizinischen Leitlinien zunehmend Aufnahme.

Für seinen außergewöhnlichen Einsatz hat Fritz Hubertus Kemper zahlreiche inund ausländische Auszeichnungen erhalten, von denen ich nur einige erwähnen möchte:

- Hartmann-Thieding-Plakette des Hartmannbundes (1989) 


\section{ZPT | nachruf}

- Paracelsus-Medaille der Bundesärztekammer (1998)

- Großes Verdienstkreuz mit Stern des Verdienstordens der Bundesrepublik Deutschland (2002)

- Ehrenpräsidentschaft (2009) und Ehrennadel der Gesellschaft für Phytotherapie (2011).
Lieber Herr Professor Kemper, lange waren Sie die Stimme der wissenschaftlichen Phytotherapie, nun sind Sie für immer verstummt. In Hochachtung vor Ihrem Lebenswerk nehmen wir traurig Abschied von Ihnen. Kein anderer hat sich national und international für die Belange der wissenschaftlichen Phytotherapie so erfolgreich eingesetzt wie Sie. Dafür gebührt Ihnen großer Dank und Anerkennung.

Die Gesellschaft für Phytotherapie wird stets ein ehrendes Andenken bewahren.

Prof. Dr. Karin Kraft

Präsidentin der Gesellschaft für

Phytotherapie 